Commun. Korean Math. Soc. 27 (2012), No. 2, pp. 425-430

http://dx.doi.org/10.4134/CKMS.2012.27.2.425

\title{
SOCIAL EQUILIBRIUM IN A GENERALIZED NASH GAME WITH THE AFFINE CONDITION
}

\author{
WON KYU KIM
}

\begin{abstract}
In this paper, we will prove a social equilibrium existence theorem of a generalized Nash game with affine constraint correspondences which is comparable with Nash's equilibrium existence theorem in several aspects.
\end{abstract}

\section{Introduction}

In mathematical economics, showing the existence of equilibrium is the main problem of investigating various kind of economic models. In general economic models, convexity assumptions are essential and basic to apply the well-known fixed point theorems as in [1-7]. Until now, there have been a number of generalized convex conditions investigated by several authors, and using those concepts, there have been numerous equilibrium existence theorems in generalized games. Recently, using Himmelberg's fixed point theorem, Kim and Lee [4] proved a new existence theorem of Nash equilibrium in a noncompact generalized game which generalizes equilibrium existence theorems due to Nash [6], and Nikaido and Isoda [7] in several aspects.

In this paper, we will prove a new social equilibrium existence theorem in a generalized Nash game with affine constraint correspondences which is comparable with an equilibrium existence theorem due to Nash [6] in several aspects.

\section{Preliminaries}

Let $I$ be a (possibly uncountable) set of players, and let $X_{i}$ be a nonempty topological space as an action space for each $i \in I$, and denote $X_{-i}:=$ $\prod_{j \in I \backslash\{i\}} X_{j}$. For an action profile $x=\left(x_{i}\right)_{i \in I} \in X=\prod_{i \in I} X_{i}$, we shall write $x_{-i}=\left(x_{1}, \ldots, x_{i-1}, x_{i+1}, \ldots\right) \in X_{-i}$; and if $x_{i} \in X_{i}, x_{-i} \in X_{-i}$, we simply write a typical strategy profile

$$
x=\left(x_{-i}, x_{i}\right):=\left(x_{1}, \ldots, x_{i-1}, x_{i}, x_{i+1}, \ldots\right) \in X_{-i} \times X_{i} .
$$

Received December 12, 2010.

2010 Mathematics Subject Classification. Primary 91A13, 52A07.

Key words and phrases. generalized Nash game, social equilibrium, affine. 
We now introduce some general notions and terminologies in generalized non-cooperative strategic games. A generalized Nash game (or social system) is an ordered triples $\Gamma=\left(X_{i} ; T_{i}, f_{i}\right)_{i \in I}$ where for each player $i \in I$, the nonempty set $X_{i}$ is a player's pure strategy space, $T_{i}: X \rightarrow 2^{X_{i}}$ is a player's constraint correspondence, and $f_{i}: X \rightarrow \mathbb{R}$ is a player's payoff (or utility) function. The set $X$, joint strategy space, is the Cartesian product of the individual strategy spaces, and the element of $X_{i}$ is called a strategy. Then, a strategy tuples $\bar{x}=\left(\bar{x}_{i}\right)_{i \in I} \in X$ is called the social equilibrium (or generalized Nash equilibrium) for the game $\Gamma$ if the following system of inequalities holds: for each $i \in I$,

$$
\bar{x}_{i} \in T_{i}(\bar{x}) \text { and } f_{i}\left(\bar{x}_{-i}, \bar{x}_{i}\right) \geq f_{i}\left(\bar{x}_{-i}, x_{i}\right) \text { for each } x_{i} \in T_{i}(\bar{x}) .
$$

When the set of players is a finite set $I=\{1, \ldots, n\}$, a generalized Nash game $\Gamma$ is called a generalized $N$-person game.

Throughout this paper, all topological spaces are assumed to be Hausdorff, and for the other standard notations and terminologies, we shall refer to $[3,4]$.

\section{Social equilibrium with affine constraint correspondences}

In a generalized Nash game with infinite players, we recall that the set of all real-valued payoff functions $\left\{f_{i} \mid i \in I\right\}$ satisfy the unconditional summability [3] if any rearrangement $\sum_{j \in I} f_{j}\left(x_{-j}, x_{j}\right)$ of the infinite sum $\sum_{i \in I} f_{i}\left(x_{-i}, x_{i}\right)$ converges to the same real value. Indeed, the unconditional summability should be needed for a generalized Nash game with infinite players, and it should be noted that $\left\{f_{i} \mid i \in I\right\}$ is unconditionally summable if the sum $\sum_{i \in I} f_{i}\left(x_{-i}, x_{i}\right)$ converges absolutely. From now on, we may assume that the set of all realvalued payoff functions $\left\{f_{i} \mid i \in I\right\}$ satisfies the unconditionally summablity in a generalized Nash game $\Gamma$.

For the social equilibrium in the generalized Nash game $\Gamma$ having the unconditionally summablity, let us define the total sum of payoff function $H$ : $X \times X \rightarrow \mathbb{R}$ associated with the strategic game $\Gamma$ as follows:

$$
H(y, x):=\sum_{i \in I} f_{i}\left(x_{-i}, y_{i}\right) \quad \text { for each } x, y \in X=\prod_{i \in I} X_{i} .
$$

Then we can obtain the following equivalences which generalizes the theorems due to $\mathrm{Lu}[5]$, and Nikaido and Isoda [7]:

Lemma 1. Let $\Gamma=\left(X_{i} ; T_{i}, f_{i}\right)_{i \in I}$ be a generalized Nash game of normal form where $I$ be a (possibly uncountable) set of players. Then the followings are equivalent:

(1) $\bar{x} \in X=\prod_{i \in I} X_{i}$ is a social equilibrium for $\Gamma$;

(2) for each $i \in I, \bar{x}_{i} \in T_{i}(\bar{x})$, and $f_{i}\left(\bar{x}_{-i}, \bar{x}_{i}\right) \geq f_{i}\left(\bar{x}_{-i}, x_{i}\right)$ for all $x_{i} \in T_{i}(\bar{x})$;

(3) for each $i \in I, \bar{x}_{i} \in T_{i}(\bar{x})$, and $H(\bar{x}, \bar{x}) \geq H(y, \bar{x})$ for all $y \in \Pi_{i \in I} T_{i}(\bar{x})$. 
Proof. The equivalence of (1) and (2) follows immediately from the definition of a social equilibrium in case of the infinite set of players. By using the unconditional summablity of $\left\{f_{i} \mid i \in I\right\}$, the implication $(2) \Rightarrow(3)$ is obtained by adding both sides of the inequalities $f_{i}\left(\bar{x}_{-i}, \bar{x}_{i}\right) \geq f_{i}\left(\bar{x}_{-i}, x_{i}\right)$ for all $i \in I$. To prove (3) implies (2), we first fix $i$, and take $y=\left(\bar{x}_{-i}, y_{i}\right)$ where $y_{i} \in T_{i}(\bar{x})$. Then the inequality $H(\bar{x}, \bar{x}) \geq H(y, \bar{x})$ may be written as

$$
f_{i}\left(\bar{x}_{-i}, \bar{x}_{i}\right)-f_{i}\left(\bar{x}_{-i}, y_{i}\right)+\sum_{j \neq i}\left(f_{j}\left(\bar{x}_{-j}, \bar{x}_{j}\right)-f_{j}\left(\bar{x}_{-j}, y_{j}\right)\right) \geq 0 .
$$

Since $y_{j}=\bar{x}_{j}$ whenever $j \neq i$, we have that for all $i \in I, f_{i}\left(\bar{x}_{-i}, \bar{x}_{i}\right)-$ $f_{i}\left(\bar{x}_{-i}, y_{i}\right) \geq 0$, which proves $(2)$.

Remark. Lemma 1 generalizes the previous results on the total sum of payoff functions due to $\mathrm{Lu}$ [5], Nash [6], and Nikaido and Isoda [7] in the following aspects:

(i) the set $I$ of players need not be a finite set;

(ii) the inequalities on $H(y, x)$ need not satisfy in the whole strategy set $X_{i}$, but on the $i$-th player's constraint set $T_{i}(\bar{x})$. In fact, when the set $I$ of players is a finite set, and $T_{i}(\bar{x})=X_{i}$ for each $i \in I$, Lemma 1 reduces to the lemma due to Nikaido and Isoda [7].

In a generalized Nash game $\Gamma=\left(X_{i} ; T_{i}, f_{i}\right)_{i \in I}$, we denote the fixed point set $\mathcal{F} \subseteq X$ of the correspondence $T=\prod_{i \in I} T_{i}: X \rightarrow 2^{X}$ by

$$
\mathcal{F}=\left\{x \in X \mid x_{i} \in T_{i}(x) \text { for all } i \in I\right\},
$$

and the range of $T$ by $\mathcal{R}(T)$.

We now prove a new existence theorem of social equilibrium for a generalized Nash game with affine constraint correspondences by applying Himmelberg's fixed point theorem as follows:

Theorem 1. Let $\Gamma=\left(X_{i} ; T_{i}, f_{i}\right)_{i \in I}$ be a non-cooperative generalized Nash game, where $I$ be a (possibly uncountable) set of players, such that for each $i \in I$, the strategy set $X_{i}$ is a convex subset in a locally convex Hausdorff topological vector space and $D_{i}$ is a nonempty compact subset of $X_{i}$. Let $X=$ $\prod_{i \in I} X_{i}$, and $D=\prod_{i \in I} D_{i}$. For each $i \in I, f_{i}: X \rightarrow \mathbb{R}$ is a player's payoff function, and $T_{i}: X \rightarrow 2^{D_{i}}$ is upper semicontinuous such that each $T_{i}(x)$ is a nonempty closed convex subset of $D_{i}$, and satisfies the affine condition that for each $\lambda \in[0,1]$,

$$
\lambda T_{i}(x)+(1-\lambda) T_{i}(y) \subseteq T_{i}(\lambda x+(1-\lambda) y) \quad \text { for all } x, y \in X .
$$

Assume that the total sum of payoff functions $H: X \times X \rightarrow \mathbb{R}$ satisfy the following conditions:

(1) $(x, y) \mapsto H(y, x)-H(x, x)$ is lower semicontinuous in $X \times X$;

(2) for each $x \in \mathcal{F},\{y \in \mathcal{R}(T) \mid H(x, x)<H(y, x)\}$ is convex in $\mathcal{R}(T)$;

(3) for each $y \in \mathcal{R}(T),\{x \in \mathcal{F} \mid H(y, x) \leq H(x, x)\}$ is nonempty convex. 
Then the generalized Nash game $\Gamma$ has a social equilibrium $\bar{x} \in X$, i.e., for each $i \in I, \bar{x}_{i} \in T_{i}(\bar{x})$, and $f_{i}\left(\bar{x}_{-i}, \bar{x}_{i}\right) \geq f_{i}\left(\bar{x}_{-i}, x_{i}\right)$ for each $x_{i} \in T_{i}(\bar{x})$.

Proof. Suppose the contrary, i.e., we assume that $\Gamma$ has no social equilibrium. Then, by Lemma $1(3)$, we have

(*) for each $x \in X$, either $x_{i} \notin T_{i}(x)$ for some $i \in I$, or there exists $y \in T(x)$ such that $H(x, x)<H(y, x)$.

Since each $T_{i}$ is upper semicontinuous having nonempty compact convex values, the correspondence $T: X \rightarrow 2^{D}$, defined by $T(x):=\Pi_{i \in I} T_{i}(x)$ for each $x \in X$, is also upper semicontinuous having nonempty compact convex values. Therefore, by Himmelberg's fixed point theorem, there exists a fixed point $\hat{x} \in X$ for $T$, i.e., $\hat{x}_{i} \in T_{i}(\hat{x})$ for each $i \in I$. Then the fixed point set $\mathcal{F} \subseteq X$ of the correspondence $T$ is a nonempty closed subset of $D$ by the upper semicontinuity of $T$. Moreover, by the affine assumption on $T_{i}$, we have $\mathcal{R}(T)$ is a convex set. Indeed, if $y_{1}, y_{2} \in \mathcal{R}(T)$ and $\lambda \in[0,1]$ are arbitrarily given, then there exist $x_{1}, x_{2} \in X$ such that $y_{1} \in T\left(x_{1}\right)$ and $y_{2} \in T\left(x_{2}\right)$. Let $x=\lambda x_{1}+(1-\lambda) x_{2} \in X$ and $y=\lambda y_{1}+(1-\lambda) y_{2} \in X$. Then for each $i \in I$,

$$
\begin{aligned}
y_{i}=\lambda\left(y_{1}\right)_{i}+(1-\lambda)\left(y_{2}\right)_{i} & \in \lambda T_{i}\left(x_{1}\right)+(1-\lambda) T_{i}\left(x_{2}\right) \\
& \subseteq T_{i}\left(\lambda x_{1}+(1-\lambda) x_{2}\right)=T_{i}(x) ;
\end{aligned}
$$

so that $\mathcal{R}(T)$ is convex. Similarly, by the affine assumption on $T_{i}$, we can also have that $\mathcal{F}$ is a convex subset of $X$.

We shall assume that for each $x \in X, x \in \mathcal{F}$ in the statement $(*)$ of the reduction ad absurdam. Then, since $x_{i} \in T_{i}(x)$ for all $i \in I$, there exists $y \in T(x) \subset \mathcal{R}(T)$ such that $H(x, x)<H(y, x)$.

For each $y \in \mathcal{R}(T)$, we let

$$
N(y):=\{x \in \mathcal{F} \mid H(x, x)<H(y, x)\} .
$$

By the assumption (1), each $N(y)$ is (possibly empty) open in $\mathcal{F}$ and we have $\bigcup_{y \in \mathcal{R}(T)} N(y)=\mathcal{F}$. Since $\mathcal{F}$ is compact, there exists a finite number of points $\left\{y_{1}, \ldots, y_{n}\right\} \subset \mathcal{R}(T)$, and nonempty open sets $N\left(y_{1}\right), \ldots, N\left(y_{n}\right)$ such that $\bigcup_{i=1}^{n} N\left(y_{i}\right)=\mathcal{F}$. Let $\left\{\alpha_{i} \mid i=1, \ldots, n\right\}$ be the continuous partition of unity subordinated to the open covering $\left\{N\left(y_{i}\right) \mid i=1, \ldots, n\right\}$ of the compact set $\mathcal{F}$, i.e.,

$$
0 \leq \alpha_{i}(x) \leq 1, \quad \sum_{i=1}^{n} \alpha_{i}(x)=1 \text { for all } x \in \mathcal{F}
$$

and if $x \notin N\left(y_{i}\right)$, for some $1 \leq i \leq n$, then $\alpha_{i}(x)=0$.

We now define a mapping $\phi: \mathcal{F} \rightarrow \mathcal{R}(T)$ by

$$
\phi(x):=\sum_{i=1}^{n} \alpha_{i}(x) y_{i} \quad \text { for each } \quad x \in \mathcal{F} .
$$

Then, $\phi$ is a continuous mapping since each $\alpha_{i}$ is continuous. By the assumption (2), for fixed $x \in \mathcal{F}$, the set $\{y \in \mathcal{R}(T) \mid H(x, x)<H(y, x)\}$ is convex in $\mathcal{R}(T)$ 
so that we have

$$
\begin{aligned}
\phi(x) & \in \operatorname{co}\left\{y_{i} \in \mathcal{R}(T) \mid \alpha_{i}(x) \neq 0\right\} \\
& \subseteq\{y \in \mathcal{R}(T) \mid H(x, x)<H(y, x)\} ;
\end{aligned}
$$

and hence we also see that $\phi$ maps $\mathcal{F}$ into $\mathcal{R}(T)$.

Next, we define a multimap $S: \mathcal{R}(T) \rightarrow 2^{\mathcal{F}}$ by

$$
S(y):=\{x \in \mathcal{F} \mid H(y, x) \leq H(x, x)\} \quad \text { for each } y \in \mathcal{R}(T) .
$$

Then, for each $y \in \mathcal{R}(T)$, by the assumption (3), $S(y)$ is a nonempty convex set in $\mathcal{F}$. By the assumption (1) again, the set $\{(x, y) \in \mathcal{F} \times \mathcal{F} \mid H(y, x)-H(x, x) \leq$ $0\}$ is non-empty closed in $\mathcal{F} \times \mathcal{F}$ and hence it is compact. Therefore, for each $y \in \mathcal{R}(T), S(y)$ is the projection of a nonempty compact set in $\mathcal{F} \times \mathcal{F}$ so that $S(y)$ is compact. Next, it is easy to see that $S$ has a closed graph in $\mathcal{R}(T) \times \mathcal{F}$. In fact, for any nets $\left(x_{\alpha}\right) \rightarrow x_{o},\left(y_{\alpha}\right) \rightarrow y_{o}, y_{\alpha} \in S\left(x_{\alpha}\right)$, we have $H\left(y_{\alpha}, x_{\alpha}\right) \leq H\left(x_{\alpha}, x_{\alpha}\right)$. Since the mapping $(x, y) \mapsto H(y, x)-H(x, x)$ is lower semicontinuous, we have $H\left(y_{o}, x_{o}\right) \leq H\left(x_{o}, x_{o}\right)$. Hence $y_{o} \in S\left(x_{o}\right)$ and $S$ has a closed graph in $\mathcal{R}(T) \times \mathcal{F}$.

Finally, we define a multimap $\Phi: \mathcal{F} \rightarrow 2^{\mathcal{F}}$ by

$$
\Phi(x)=(S \circ \phi)(x) \quad \text { for each } \quad x \in \mathcal{F} .
$$

Since $S$ has a closed graph and $\phi$ is continuous, $\Phi$ has a closed graph in a compact set $\mathcal{F} \times \mathcal{F}$, and each $\Phi(x)$ is nonempty compact convex. Therefore, by the Fan-Glicksberg fixed point theorem, there exists a fixed point $\bar{x} \in \mathcal{F}$ for $\Phi$ such that $\bar{x} \in \Phi(\bar{x})=S(\phi(\bar{x}))$. Let $x^{*}=\phi(\bar{x}) \in \mathcal{R}(T)$; then

$$
\bar{x} \in S\left(x^{*}\right)=\left\{x \in \mathcal{F} \mid H\left(x^{*}, x\right) \leq H(x, x)\right\}
$$

so that $H\left(x^{*}, \bar{x}\right) \leq H(\bar{x}, \bar{x})$. On the other hand, since $x^{*}=\phi(\bar{x})$, by the inclusion $(\dagger)$, we have

$$
x^{*}=\phi(\bar{x}) \in\{y \in \mathcal{R}(T) \mid H(\bar{x}, \bar{x})<H(y, \bar{x})\}
$$

so that $H\left(x^{*}, \bar{x}\right)>H(\bar{x}, \bar{x})$, which is a contradiction. This completes the proof.

Remark. Theorem 1 is a new equilibrium existence theorem which is comparable with the previous existence theorems of Nash equilibrium due to Lu [5], Nash [6], and Becker and Damianov [1] in the following aspects:

(i) the set $I$ of players need not be a finite set;

(ii) the strategy set $X_{i}$ need not be a compact set;

(iii) every payoff function $f_{i}$ need not be (quasi)concave nor continuous on $X$. Indeed, when $T_{i}(x)=X_{i}=D_{i}$ for each $i \in I$, then we have $\mathcal{R}(T)=\mathcal{F}=X$ so that if each $f_{i}: X \rightarrow \mathbb{R}$ is continuous on $X$ and the function $y_{i} \mapsto f_{i}\left(x_{-i}, y_{i}\right)$ is quasiconcave on $X_{i}$ as in [5, 6,7], then the assumptions (1) and (2) of Theorem 1 are satisfied. 
As remarked, when $X_{i}=D_{i}$ is compact and convex, if we let $T_{i}(x):=X_{i}$ for each $x \in X$ and $i \in I$, then we have $\mathcal{R}(T)=\mathcal{F}=X$ and $T_{i}$ automatically satisfies the affine condition so that we can obtain the following:

Theorem 2. Let $\Gamma=\left(X_{i} ; f_{i}\right)_{i \in I}$ be a non-cooperative strategic game of normal form, where $I$ be a (possibly uncountable) set of players, with the strategy set $X_{i}$ being nonempty compact convex subset in a locally convex Hausdorff topological vector space $E, X=\Pi_{i \in I} X_{i}$, and $f_{i}: X \rightarrow \mathbb{R}$ being player's payoff function. Assume that the total sum of payoff function $H: X \times X \rightarrow \mathbb{R}$ satisfy the following conditions:

(1) $(x, y) \mapsto H(y, x)-H(x, x)$ is lower semicontinuous on $X$;

(2) for each $x \in X,\{y \in X \mid H(x, x)<H(y, x)\}$ is convex in $X$;

(3) for each $y \in X,\{x \in X \mid H(y, x) \leq H(x, x)\}$ is convex.

Then the infinite strategic game $\Gamma$ has a Nash equilibrium.

Acknowledgments. This research was supported by Basic Science Research Program through the National Research Foundation of Korea(NRF) funded by the Ministry of Education, Science and Technology (No. 2010-0009386), and the author thanks the referee for his valuable suggestions for improvement of the paper.

\section{References}

[1] J. G. Becker and D. S. Damianov, On the existence of symmetric mixed strategy equilibria, Econom. Letters 90 (2006), no. 1, 84-87.

[2] G. Debreu, A social equilibrium existence theorem, Proc. Nat. Acad. Sci. U.S.A. 38 (1952), 386-393.

[3] W. K. Kim and S. Kum, Existence of Nash equilibrium in a compact acyclic strategic game, J. Chungcheong Math. Soc. 23 (2010), 29-35.

[4] W. K. Kim and K. H. Lee, Nash equilibrium and minimax theorem with $\mathcal{C}$-concavity, J. Math. Anal. Appl. 328 (2007), no. 2, 1206-1216.

[5] H. Lu, On the existence of pure strategy Nash equilibrium, Econom. Letters 94 (2007), no. 3, 459-462.

[6] J. Nash, Non-cooperative games, Ann. Math. 54 (1951), 286 - 295.

[7] H. Nikaido and K. Isoda, Note on non-cooperative convex games, Pacific J. Math. 5 (1955), $807-815$.

Department of Mathematics Education

Chungbuk National University

Cheonguu 361-763, KoreA

E-mail address: wkkim@chungbuk.ac.kr 